\title{
Congenital Heart Disease Patient Home Care Services Using the Interactive TV: The PANACEIA-ITV Approach
}

\author{
N Maglaveras ${ }^{1}$, I Lekka ${ }^{1}$, I Chouvarda ${ }^{1}$, V Koutkias $^{1}$, M Gatzoulis $^{2}$, T Kotis ${ }^{2}$, M Tsakali $^{3}$, S Maglavera ${ }^{4}$, \\ V Danelli ${ }^{5}$, B Zeevi ${ }^{6}$, EA Balas ${ }^{7}$ \\ ${ }^{1}$ Lab of Medical Informatics, Aristotle University, Thessaloniki, Greece, ${ }^{2}$ Brompton Royal Hospital, \\ London, UK, ${ }^{3}$ INTRACOM S.A. Athens, Greece, ${ }^{4}$ Pouliadis Associates, Thessaloniki, Greece, ${ }^{5}$ OTE \\ S.A., Athens, Greece, ${ }^{6}$ Card Guard, Scientific Survival, Rehovot, Israel, ${ }^{7}$ School of Public Health St. \\ Louis University, St. Louis, MO, USA
}

\begin{abstract}
In the context of the PANACEIA-ITV IST European project, a home care service provision system is described based on interactive TV technology. The purpose of PANACEIA-ITV is to facilitate essential lifestyle changes and to promo te compliance with scientifically sound selfcare recommendations, through the application of interactive digital television for family health maintenance. The means to achieve these goals are based on technological, health services and business models. PANACEIA-ITV is looking for communication of monitoring micro-devices with HTV set-top-boxes using infrared technology, and embodiment of analogous $H / W$ and $S / W$ in the ITV set-top-boxes. The PANACEIA-ITV system is used by patients suffering from adult congenital heart disease (ACHD) at the Royal Brompton Hospital. The primary aim of this pilot is to test whether delivery of high quality monitoring, treatment and education for the ACHD or pulmonary hypertension patient is feasible via interactive television and infrared microdevices. Secondary aims of this pilot are to test the hypothesis that intensified home monitoring and guidance for patients of this target group will lead to reduced ACHD related hospitalization and to evaluate the overall attitude of the patients and clinicians towards the use of new technologies such as ITV in healthcare and disease management.
\end{abstract}

\section{Introduction}

Information technology (IT) applications in medicine are rapidly expanding and new methods and solutions are evolving, since they are considered pivotal in the success of preventive medicine [1]. In the past days, IT applications were scarce, difficult to use, maintain, and expensive. Today, due to the fast growing and penetration of the INTERNET and mobile telephone technology, the IT applications in the health care environment are focused at e-consultation [2], home care delivery [3] and the use of triage systems [4]. Home care delivery is a very important issue, covering the management of chronic diseases, wellness, education and information delivery on-demand, and addressing socially delicate targeted problems such as infertility. Thus, there is a demand for continuous monitoring of basic parameters and biosignals of the patient, as well as timely delivery of educational material, for prevention of complications and the advent of serious diseases. One of the most important IT areas where work needs to be done is the interactive TV (ITV) area, since TV is a communication medium accessible to all citizens and to all houses that can increase regional health quality [5].

In this paper, the use of ITV for Citizen Centered Health and Lifestyle Management system is considered, by use of the PANACEIA-ITV paradigm. The PANACEIAITV system is used by patients suffering from adult congenital heart disease (ACHD) at the Royal Brompton Hospital. The primary aim of this pilot is to test whether delivery of high quality monitoring, treatment and education for the ACHD or pulmonary hypertension patient is feasible via interactive television and infrared microdevices.

\section{Methods}

The communication means used in this work are satellite TV for the patient and Internet for the clinician. Patient needs a very simple interface that will guide him/her through the different services provided and will be 
handled with the remote control. Clinician needs a more sophisticated interface for patient monitoring.

At the end users' environment the Patient's interface is managed through the STB, which communicates via an IR link with the medical microdevices. The patient is able to interact with the PANACEIA-ITV service provider receiving Educational Videos, Messages and other patient related info, either over satellite transmission or via the Return Channel. Patient Data, entered with a Remote Control Unit and the medical microdevices, are submitted to the PANACEIA-ITV Server via the Return Channel.

The PANACEIA-ITV system is modular, consisting of front-end and back-end modules responsible for accomplishing specific tasks. Web interfaces are provided to the Contact Center, the Clinicians and the Educational Material Providers. Databases exist for storing patients' profiles and clinical data, educational material related info and profile info of the rest of the PANACEIA-ITV actors.

In the following sections, the PANACEIA-ITV system is described in more detail from a functional and technical point of view.

\subsection{Scenarios of use}

As far as the target groups are concerned, PANACEIAITV starts with specific patient and general population groups, which will define the superset of user requirements and will evaluate the PANACEIA-ITV platform. One of the scenarios of use is Congenital Heart Disease patients.

Users and Stakeholders have been defined for the system. The Users of the system are Patients, Clinicians and Contact Center personnel. The Stakeholders are the Technology Providers, e.g. Broadcaster, Medical Device Manufacturers, Educational Material Providers. In the following, each role is described in more detail.

Patients: Users at home may use the PANACEIA-ITV services such as data exchange, prompting and querying for communicating with the medical professionals through their TV sets and STBs. An important aspect is that users at home are able to receive educational material in the form of tips, digital videos or interactive hypertext.

Clinicians, i.e. Doctors \& Nurses: The clinicians are responsible for monitoring their patients' condition by checking the transmitted measurements and scheduling the patient's activities and communication protocol via the PANACEIA-ITV system. Using a customized web interface they are able to view the values of the measurements that the patients perform at home, check biosignals and check patients' schedule compliance. Finally, medical staff is responsible for reviewing any educational material submitted for broadcasting.
Contact Center: The main role of the Contact Center staff is to administrate the PANACEIA-ITV application and specifically all the patients that are registered with the PANACEIA-ITV service, register new patients and train them, receive the educational multimedia material from the Educational Material Provider, define its scheduling and deliver it to the broadcaster.

Educational Material Providers: They provide the educational material to the PANACEIA-ITV system, in the form of tips, hypertext/images or videos. The educational material is associated with specific patient groups and group categories.

Broadcaster: Corresponds to the owner of the digital broadcasting platform and its role is to receive the content and the ITV applications and to broadcast them to the registered households with the PANACEIA-ITV Service.

\subsection{System description}

PANACEIA-ITV is a multi-component system. The main innovation lies in the integration of different modules and technologies (Figure 1).

PANACEIA-ITV Front-End: Patients' STB receives and processes the broadcasted data (audio/video plus the PANACEIA-ITV application). The STB executes the application according to the user's profile. This scheme allows the user to interact with the application and communicate with the PANACEIA-ITV Server (full twoway interaction). Furthermore, it allows IR communication between the STB and the medical microdevices. The equipment available for the patient will be the STB, the microdevices and the Remote Control. IR communication with the STB is mandatory for signal and vital parameter transmitting microdevices, such as the 12-lead ECG.

Broadcaster: The Broadcaster owns bandwidth on a broadband network and possesses the infrastructure for broadcasting. This part is assigned the task to schedule events and allocate bandwidth for the transmission of educational video and audio streams. Cable, satellite or terrestrial transmission may be used.

PANACEIA-ITV Server: It controls and provides communication links between the different PANACEIAITV users, mainly by regulating the information and request flow, by aggregating content and service modules for multiple modality access, and by incorporating the distributed databases holding educational material and citizen/patient data.

Application Server: The PANACEIA-ITV Application Server is a component of the PANACEIA back-end. It holds the executables and the data of the target platform and schedules them for delivery to the network operator. It 
handles HTTP requests coming from the STB. Periodically, it receives content from the Portal, converts it to the appropriate format and delivers it to the broadcaster.

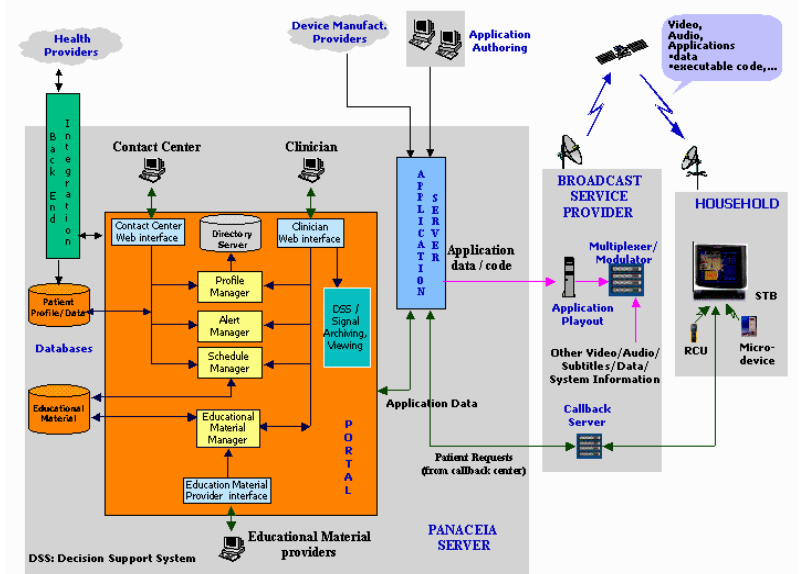

Figure 1. The PANACEIA-ITV system architecture.

Portal: The Portal provides Membership Management, i.e. the creation/management of Users, Groups, Roles and Domains. It also provides integration services allowing deployment of WWW applications. It has mechanisms for reporting/accounting/logging, as well as for handling of all data requests.

Multi-Agent System: Software agents provide additional intelligence and advanced functionalities to the Medical Personnel [6]. The agent-based systems incorporated in PANACEIA-ITV are:

- The Alert Manager, which notifies the clinicians of non-trivial situations related to patients' measurements.

- The Schedule Manager that checks the patient's compliance to the pre-planned activities and provides suggestions to the clinicians for plan adaptations by evaluating patient's medical values.

- The Educational Material Manager, which assists the Contact Center personnel in educational content management.

DSS/Signal Archiving and processing: Decision Support System (DSS) in PANACEIA-ITV identifies patients whose data follow specific patterns. First, data mining mechanisms extract rules from the stored patient data. Second, a rule editor allows clinicians to apply the extracted rules on any patient dataset or create their own. The Signal Archiving and Processing module includes archiving mechanisms and viewers for ECG signals. Signal processing and interpretation (denoising, filtering, information extraction) tools are also available.
Databases: The PANACEIA-ITV Databases have an expandable and parametric structure. In the Patient Database, patient's demographic data, medical data and details about the services provided to the patient are stored, while sessions of educational videos, text and short textual tips are stored in the Educational Material Database. The Portal resources ae used for data exchange between the Databases and various applications.

\subsection{Clinical trial on ACHD patients}

ACHD (Adult Congenital Heart Disease) patients who will participate at the PANACEIA-ITV pilot will follow a specific treatment plan. They will transmit measurements at prescribed intervals, through the PANACEIA-ITV channel at their homes. Specifically, twice a week they will record and transmit body weight, heart rate, blood pressure, oxygen saturation and they will answer to two symptoms and sign questions. Once in two weeks, they will record and transmit their 12 lead ECG, via an ECG microdevice that communicates via Infrared with the TV. Initial filtering of data will produce appropriate messages to the patients (cases of erroneous data, emergencies where patient has to directly contact the hospital).

The clinician on the Royal Brompton side will be monitoring the transmitted data and, when necessary, he/she will send to the patient messages with recommended actions (change in medication dosage, prompt for hospital visit, etc). Patients will view these messages as soon as they login on the PANACEIA-ITV channel. In addition, they will be able to view at any time, their prescribed medications as well as their scheduled clinical actions (hospital visits, measurement transmission, and lab tests). Finally, they can see the progress of their condition by reviewing their transmitted data over a period of time together with the clinician's comments. Important information and advice regarding their condition will be also available at any time on their TV. Moreover, lab tests and outpatient clinic visits will be done in prescribed intervals, according to their condition. It has to be emphasized that PANACEIA-ITV offers intensified monitoring but does not aim to cover emergency situations. Patients will still have to directly contact the hospital, as they have always been doing, whenever there is an emergency.

\section{Results}

When the first User Interface (UI) prototype for patients was available (Figure 2a), a usability test with real users was performed. The user group consisted of 6 patients, 3 citizens interested in managing their health and 2 
clinicians. The method followed to demonstrate the application to users was to have the prototype running on a regular TV that was connected to the PANACEIA-ITV STB. All interactions were done via the STB's remote control.

First, the clinicians tested the prototype in order to check the compliance with the user requirements. Then, each user tested the system separately with the assistance of a facilitator. The facilitator gave instructions of how to use PANACEIA-ITV and the user then received the remote control and tried to do the same. The user spoke freely during this process and the facilitator documented all comments. The session closed with an interview where the facilitator asked questions not only on the interface and system use but also on the general usage of such systems for health management. The whole session lasted for about $1.5 \mathrm{hr}$ for each patient/citizen.

The overall rating of the system was high. All 9 users (patients and citizens) were very satisfied with the prototype, the majority of them stating that they would use the service; or that they were very eager to participate to the pilot. All users would recommend it to family and friends, they were interested in the educational part and found the use of the IR microdevices useful, but their main concerns were the cost, how often they would get response from the clinicians and whether established clinical institutions would be providing the clinical service.

\section{Conclusions}

In the current paper, functional and technical aspects of the PANACEIA-ITV system are presented. It consists of a feasibility study on the application of ITV technology for home care services. So far, the preliminary tests show that the proposed system has prospects to penetrate the home care market offering the possibility for quality health care services, and increased patient safety. The intelligence of the system lies in the use of multi-agent systems performing complex tasks and the service provision model, following the Active Service Provision (ASP) approach. Clinicians can use WWW interfaces for browsing of biosignals and other biodata (Figure 2b), and these interfaces are available via the WWW and the ITV platform as well, offering enormous and limitless possibilities for disease management.

\section{Acknowledgements}

This work is part of the project PANACEIA-ITV (IST2001-33369) funded by the European Commission.

\section{References}

[1] Collen MF. Historical evolution of preventive medical informatics in the USA. Meth Inform Med 2000;39(3):204-7.

[2] Borowitz SM, Wyatt J. The origin, content, and workload of E-mail consultations. JAMA 1998;280:1321-4.

[3] Balas EA, Iakovidis I. Distance technologies for patient monitoring. BMJ 1999;319:1309.

[4] Rosenblatt E. Telephone triage. A common sense approach. RN 2001;64(3):suppl 2-3.

[5] Maglaveras N, Chouvarda I, Koutkias V, Meletiadis S, Haris K, Balas EA. Information technology can enhance quality in regional health delivery. Meth Inform in Med 2002;41(5):393-400.

[6] Koutkias V, Chouvarda I, Maglaveras N. Agent-based Monitoring and Alert Generation for a Home Care Telemedicine System. In Proc. AMIA 2002:395-399.

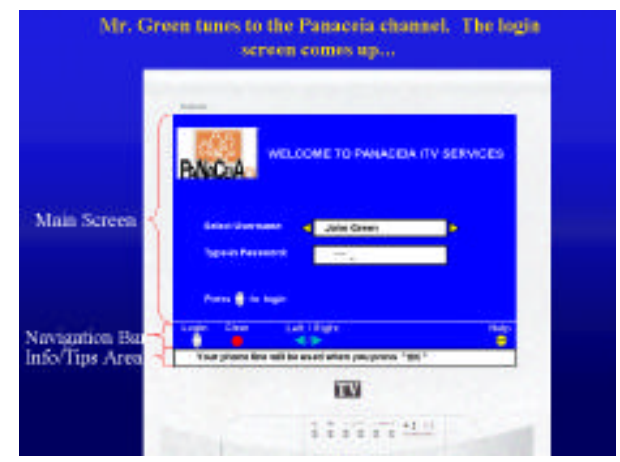

(a)

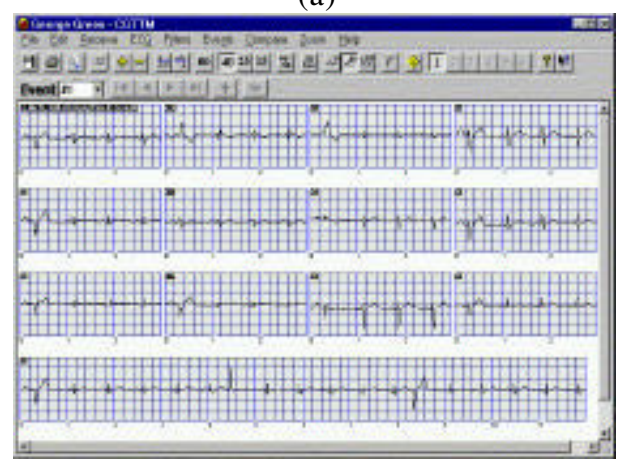

(b)

Figure 2. PANACEIA-ITV user interfaces: (a) Patient interface (TV) and (b) 12 lead ECG Clinician interface.

Address for correspondence: Nicos Maglaveras, $\mathrm{PhD}$, Associate Professor, Aristotle University, The Medical School, Lab. of Medical Informatics - Box 323, 54124 Thessaloniki, GREECE, EMAIL : nicmag@med.auth.gr 